\title{
Using Neural Networks for the Extraction of Built-Up Areas from Sentinel-2
}

\author{
Lucie Orlíková ${ }^{+}$ \\ VSB - Technical University of Ostrava, Czech Republic
}

\begin{abstract}
As urban areas develop, changes occur in their landscape. Cities or metropolitan areas are typically warmer with slightly higher temperatures compared to their adjacent rural areas. This temperature difference is due to the unusual state known as the urban heat island. Buildings, roads and other infrastructure replace open land and vegetation. Detecting urban areas from remote sensing images plays an important role in the field of Earth observation. In this paper, we propose to exploit the benefit of Sentinel-2 images to extract built-up areas. The following machine learning methods have been tested: random forests, support vector machines and multi layered perceptron. Experiments are performed in Ostrava area, in the Czech Republic. The validation is carried out using a control dataset which is Corine Land Cover 2012. The results were validated by a Kappa index and it showed that random forests is among the best performing method for the classification. The main result obtained in this paper is that the neural network considered here provides a satisfying effect for the classification of urban multispectral images.
\end{abstract}

Keywords: Urban areas, Sentinel 2, Land cover, Machine learning, Neural networks

\section{Introduction}

Over the last few decades, urban area detection from remote sensing images has become more important for many applications, such as urban planning, health monitoring, ecology, map updating, disaster management etc. As urban areas develop, changes occur in their landscape. Surfaces that were once permeable and wet become impermeable and dry. These changes cause urban areas to become warmer than their rural surroundings, forming an "island" of higher temperatures in the landscape [2].

Understanding the urban growth phenomenon and the effects of artificial surfaces to surface and atmospheric temperature are among the major issues that public services have to deal with. Local governments can take steps to help residents, infrastructure, and systems reduce their vulnerability to heat, both in response to an extreme heat event and as part of longer-term planning to lessen future risks. To reduce the heat island effect and its associated risks over the longer term, communities can use four main strategies: increasing tree and vegetative cover, creating green roofs, installing cool-mainly reflective roofs and using cool pavements [11].

One of the main goals of remote sensing is to label images according to a set of semantic categories, i.e. image classification. So recently deep learning has become the new state-of-the-art solution for image processing. Improving the accuracy of land cover classification has attracted a great deal of recent interest in remote sensing studies. Detailed and accurate classification of built-up areas is one of the basic problems, arising out of the enormous spatial and spectral heterogeneity of urban areas, in which built-up structures (buildings, transportation areas), various types of vegetation cover (e.g. parks, gardens, agricultural areas), bare soil zones and bodies of water exist in close proximity [6].

The launch of the Sentinel-2A and Sentinel-2B satellites makes available data with a minimum spatial resolution of 10 metres, 13 spectral bands, wide acquisition coverage and short time revisits, which opens a

\footnotetext{
${ }^{+}$Corresponding author. Tel.: + 420777310656.
}

E-mail address: lucie.orlikova@vsb.cz. 
large scale of new applications [9]. The European Commission conducts projects, based on remote sensing data, to monitor urban areas in order to serve regional funding and policy making. One of the objectives of the Copernicus program developed by the European Commission is to provide information products for monitoring the growth of urban areas in order to assess whether policies to reduce urban sprawl are effective.

There is a vast literature on classification approaches that consider the spectrum of every individual pixel to assign it to a certain class. In the content of spectral classification, decision trees [6], artificial neural networks $[1,12,13,15]$ and support vector machines are some of the approaches that have been explored, both for multispectral and hyperspectral image analysis [8].

The goal of this paper is to analyse the performance of the different machine learning algorithms for land-cover mapping using a Sentinel-2 images. In the following section is a study area described. The available data and detailed description of the methods are presented in Section 3. The classification results and discussion appear in Section 4. The main conclusions appear in Section 5.

\section{Study Area and Data}

Ostrava is the third largest city in the Czech Republic with around 300 thousand inhabitants and the administrative centre of the North Moravian-Silesian region. Ostrava grew to prominence thanks to its position at the heart of a major coalfield, becoming an important industrial center. It used to be nicknamed the country's "steel heart" thanks to its status as a coal-mining and metallurgical center.

The area is roughly composed of urban areas, grassland and crop sowable areas. In this study, our approach is evaluated on the Sentinel-2 dataset, that consists of 2 multispectral sub-images acquired over the area of Ostrava, on $11^{\text {th }}$ of August 2017. The Sentinel-2A image used in this manuscript is level 1C product, which is radiometrically and geometrically corrected dataset. Sentinel-2A satellite imagery consists of ten VNIR bands and three SWIR bands. The imagery produced by the Sentinel-2A satellite has spatial resolutions of 10,20 , and $60 \mathrm{~m}$. According to the Sentinel-2A data quality report, the 10th of the thirteen bands in a Sentinel-2A image is an uncalibrated band [4]. This band displays a noise pattern in bright images that are generated by detector saturation. The original image of 13 bands was cropped to the study area.

The reference set was extracted from the vector version of Corine Land Cover (CLC) product of year 2012 [3]. The positive areas for the artificial surfaces were extracted from CLC classes of continuous urban fabric (code 1.1.1), discontinuous urban fabric (code 1.1.2), industrial or commercial units (code 1.2.1) and road and rail networks and associated land (code 1.2.2). Another four land cover types were defined in the study: forest areas, agricultural areas, bare soils and water bodies.

In Fig. 1 is the area of interest in false-color composition (bands 12, 11, 4) useful for visualizing urban environments. Because this band combination makes use of the both SWIR bands aboard on Sentinel-2, the image is much more crisp than band combinations that make use of the bands in shorter wavelengths, which are more susceptible to haze.

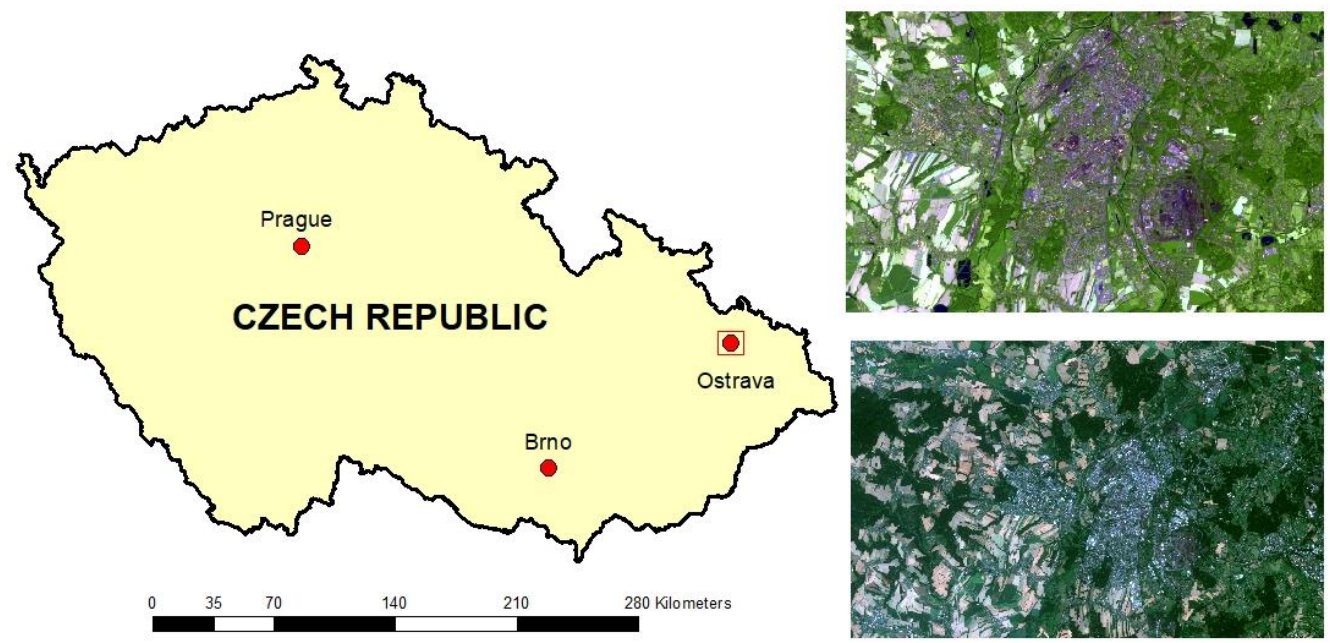

Fig. 1: Study area of Sentinel-2A imagery: a) false-color urban composite, b) true color composite 


\section{Methods Description}

Image classification is the process of assigning land cover classes to pixels. Unsupervised and supervised classification techniques are the two most common approaches. The machine learning methods testing in this study are given below:

- Multi Layered Perceptron (MLP)

- Support Vector Machine (SVM)

- Random Forest (RF)

A very brief explanation of each machine learning method is given below with some references for further reading.

The MLP algorithm is a feedforward artificial neural network model that maps sets of input data onto a set of appropriate outputs. MLP consists of a number of interconnected processing units, arranged in three layers - input layer, one or more hidden layer, and output layer. The numbers of hidden layer and the number of their units affect overall classification accuracy [5]. A network of selected architecture was trained by means of a training set of pixels to set the weights associated with neuronal interconnections. The aim of training is to build a model that can predict outputs from inputs it has never seen before.

SVM is another popular machine learning method which has been particularly applied in remote sensing by several studies $[10,17]$. In its original formulation [16] the method is presented with a set of labeled data instances and the SVM training algorithm aims to find a hyperplane that separates the dataset into a discrete predefined number of classes in a fashion consistent with the training examples. Learning refers to the iterative process of finding a classifier with optimal decision boundary to separate the training patterns (in potentially high-dimensional space) and then to separate simulation data under the same configurations (dimensions) [17].

$\mathrm{RF}$ is a very well-performing algorithm which grows many classification trees. To classify a new object form an input dataset, put the set of observations down each of the trees in the forest. Each tree gives a classification, and we say the tree votes for that class. The forest chooses the classification having the most votes. The main feature of the random forest method that makes it particularly interesting are that it is unexcelled in accuracy among current algorithms, it runs efficiently on large datasets, it can handle thousands of input variables without variable deletion and it gives estimates of what variables are important in the classification [14].

\section{Results and Discussion}

Several artificial neural network architectures for MLP method, with different numbers of input units and also hidden layers and different numbers of output units were tested. For a MLP classification results, a confusion matrix was computing. Six accuracy indices were calculated: an error of omission (EO), an error of commission (EC), consumer's accuracy (CA), producer's accuracy (PA), kappa index (KI) and overall accuracy.

Classification error occurs when a pixel belonging to one category is assigned to another category. Errors of omission occur when a feature is left out of the category being evaluated, errors of commission occur when a feature is incorrectly included in the category being evaluated. In the extraction of built-up areas, about $9 \%$ of the pixels were assigned to other class, mainly to the agricultural areas.

The consumer's accuracy is computed using the number of correctly classified pixels to the total number of pixels assigned to a category. It takes errors of commission into account by telling the consumer that, for all areas identified as category $\mathrm{X}$, a certain percentage are correct. The highest value was derived for built-up areas, $98 \%$ of pixels were assigned to them truly belong to built-up areas. This accuracy was very high for all classes except the class covering water bodies.

The producer's accuracy informs the image analyst of the number of pixels correctly classified in a category as a percentage of the total number of pixels actually belonging to that category in the image. Producer's accuracy measures errors of omission. This accuracy was highest (more than $94 \%$ of pixels) for forest, agricultural and bare soils classes. 
Another accuracy indicator is kappa coefficient. It is a measure of how the classification results compare to values assigned by chance. It can take values from 0 to 1 . If kappa coefficient equals to 0 , there is no agreement between the classified image and the reference image. If kappa coefficient equals to 1, then the classified image and the ground truth image are totally identical. So, the higher the kappa coefficient, the more accurate the classification is. The kappa coefficient for built-up class is 0.643 .

Table 1: Accuracy assessment (MLP)

\begin{tabular}{|l|c|c|c|c|c|}
\hline Class & EO $(\%)$ & EC $(\%)$ & CA $(\%)$ & PA $(\%)$ & KI \\
\hline Built-up & 9.23 & 1.37 & 98.23 & 92.45 & 0.643 \\
\hline Forest & 5.78 & 3.18 & 96.98 & 96.79 & 0.702 \\
\hline Agricultural & 3.70 & 5.15 & 95.78 & 95.47 & 0.659 \\
\hline Bare soil & 3.26 & 5.67 & 94.93 & 94.98 & 0.582 \\
\hline Water bodies & 15.10 & 85.01 & 52.97 & 84.23 & 0.426 \\
\hline
\end{tabular}

The training a SVM consists in solving the convex quadratic programming problem, for a given kernel and given value of the regularisation parameter. Three different types of SVM kernels were tested: linear kernel, polynomial kernel and radial basis kernel. The overall accuracy using the best k-NN classifier is 96.36\% for built-up areas, $97.10 \%$ for agricultural class, $96.89 \%$ for forest class, $95.87 \%$ for bare soil class and $84.29 \%$ for water bodies.

A RF combines the classifications made by many individual decision trees. To establish the optimal value of these parameters, a number of experiments were carried out using a different number of trees and a different number of split variables. The number of trees ranged between 1 and 1000 and the number of split variables was fixed from 1 to 9, using intervals of 1 . The resulting models were evaluated using the Kappa coefficient. Kappa coefficient for built-up areas was 0.87 .

\section{Conclusion}

The need for accurate and actual land cover information has become pressing. Different land cover categories contribute differently to surface and air temperature and a compilation of more precise land cover maps are very important. Three different methods were applied for the detection of urban areas from Sentinel-2 images. These artificial neural network methods can be a powerful tool for image classification. Overall, the random forest method is the best performing method for the classification. Next, SVM follows closely to random forest. SVM is used to locate optimum boundaries between classes, which in return generalize to unseen samples with least error among all possible boundaries separating two classes. The MLP neural network is the most used, it is relatively easy to use and it works fast in contrast to random forest and SVM methods. Overall accuracy of the MLP classification was almost 94\%. The results exhibit very good accuracies with Kappa coefficient over 0.6. This illustrates the ability of Sentinel-2 images, both in terms of spectral and spatial resolutions, to detect urban areas. Hence it is thought that it will be an interesting topic for future research.

\section{Acknowledgement}

Lucie Orlíková was kindly supported by VSB-Technical University of Ostrava project SV511811/SP2018/1.

\section{References}

[1] Bagan, H., Wang, Q., Watanabe, M., Kameyana, S., Bao, Y. 2008. Land-cover classification using ASTER multiband combinations based on wavelet fusion and SOM Neural Network. Photogrammetric Engineering and Remote Sensing 74(3), 333-342.

[2] Berdahl P. and S. Bretz. 1997. Preliminary survey of the solar reflectance of cool roofing materials. Energy and Buildings 25:149-158.

[3] CLC 2012 - Copernicus Land Monitoring Services: https://land.copernicus.eu/eagle/files/eagle-relatedprojects/pt_clc-conversion-to-fao-lccs3_dec2010 
[4] European Space Agency (ESA). Sentinel-2 Data Quality Report; ESA: Paris, France, 2017.

[5] FOODY, G. M. (1995): Land cover classification by an artificial neural network with ancillary information. International Journal of Geographical Information Systems 9(5), 527-542. http://dx.doi.org/10.1080/02693799508902054

[6] Herold, M., Scepan, J., Muller, A., Gunther, S. 2002. Object-oriented mapping and analysis of urban land use/cover using IKONOS data. In: 22nd Earsel Symposium Geoinformation for European-Wide Integration.

[7] Kumar, A.S., Majumder, K. 2001. Information Fusion in Tree Classifiers. Int. J. Remote Sens. Volume 22, No.5. pp. 861-869.

[8] Maggiori, E., Tarabalka, Y., Charpiat, G., Alliez, P. 2017. Convolutional Neural Networks for Large-Scale Remote Sensing Image Classification. IEEE Transactions on Geosciences and Remote Sensing. Volume 55, No.2. pp. 645-657.

[9] Malenovský, Z., Rott, H., Cihlar, J., Schaepman, M.E., García-Santos, G., Fernandes, R., Berger, M. 2012. Sentinels for science: Potential of Sentinel-1, -2, and -3 missions for scientific observations of ocean, cryosphere, and land. Remote Sensing of Environment. Volume 120, pp. 91-101.

[10] Mountrakis, G., Im, J., Ogole, C. 2001. Support Vector Machines in remote sensing: A review. ISPRS Journal of Photogrammetry and Remote Sensing. Volume 66, Issue 3. pp. 247-259.

[11] Oke, T.R. 1997. Urban Climates and Global Environmental Change. In: Thompson, R.D. and A. Perry (eds.) Applied Climatology: Principles \& Practices. New York, NY: Routledge. pp. 273-287.

[12] Pirroti, F., Sunar, F., Piragnolo, M. 2016. Benchmark of machine learning methods for classification of a Sentinel2 image. The International Archives of Photogrammetry, Remote Sensing and Spatial Information Sciences. Volume XLI-B7, XXIII ISPRS Congress, Prague, Czech Republic.

[13] Sowmya, B., Thirumaran, A., Aravindh, R., Prasad, A. A. 2011. Land cover classification using Adaptive Resonance Theory-2. In: Electronics, Communication and Computing Technologies (ICECCT), 2011 International Conference on. IEEE. pp. 78-82.

[14] Tan, Y., Ren, F., Xiong, S. 2017. Automatic extraction of built-up area based on deep convolution neural network. In. Geoscience and Remote Sensing Symposium (IGARSS), Forth Worth, USA.

[15] Tehrany, M. S., Pradhan, B., Jebu, M. N. 2013. A comparative assessment between object and pixel-based classification approaches for land use/land cover mapping using SPOT 5 imagery. Geocarto International, aheadof-print: $1-19$.

[16] Vapnik, V., 1979. Estimation of Dependences Based on Empirical Data. Nauka, Moscow, pp. 5165-5184, 27 (in Russian) (English translation: Springer Verlag, New York.

[17] Zhu, G., Blumberg, D.G., 2002. Classification using ASTER data and SVM algorithms; The case study of Beer Sheva, Israel. Remote Sensing of Environment 80 (2). pp.233-240. 